\title{
Systematic review of health state utility values for acute myeloid leukemia
}

This article was published in the following Dove Press journal:

ClinicoEconomics and Outcomes Research

\section{Anna Forsythe' \\ Patricia S Brandt ${ }^{2}$ \\ Mike Dolph' \\ Sachin Patel ${ }^{3}$ \\ Adrian Paul J Rabe' \\ Gabriel Tremblay'}

'Purple Squirrel Economics, New York, NY, ${ }^{2}$ Novartis Pharmaceuticals, East Hanover, NJ, USA; ${ }^{3}$ Novartis Pharmaceuticals UK Limited, Frimley, Camberley, Surrey, UK
Correspondence: Anna Forsythe Purple Squirrel Economics, 4 Lexington Avenue, Suite I5K, New York, NY 10010, USA

Tel +l 6464788213

Email annaforsythe@pshta.com
Background: Cost-utility analyses for acute myeloid leukemia (AML) require health state utility values (HSUVs) in order to calculate quality-adjusted life-years (QALYs) for each health state. Aim: This study reviewed AML-related HSUVs that could be used in economic evaluation studies.

Materials and methods: EMBASE, MEDLINE, and Cochrane databases were searched from January 2000 to November 2016 for relevant studies that reported quality of life (QoL) and HSUVs in AML. Identified relevant European Organization for Research and Treatment of Cancer Quality of Life Questionnaire Core 30 values were mapped to HSUVs. HSUVs for each health state in the AML treatment pathway were then collated.

Results: Ten relevant studies were identified. Six were cost-effectiveness analyses utilizing HSUVs for calculation of QALYs, one was an effectiveness analysis (incremental QALY), and two were QoL studies reporting AML-specific utilities. An additional study reported QoL for patients undergoing stem cell transplantation (SCT). Since no study reported HSUVs for relapse, values from a study of secondary AML patients who failed prior treatment for myelodysplastic syndrome were used. Where multiple HSUVs were available, collected values were given priority over assumed values. AML treatment (induction, consolidation, or SCT) was associated with decreased HSUV, while post-treatment complete remission led to increased HSUV.

Conclusion: There are some methodologically robust HSUVs that can be directly used in economic evaluations for AML. Careful interpretation is advised considering significant differences in methodologies and patient population (inclusion, size). We need to develop HSUVs with larger-sized studies, making greater use of condition-specific data.

Keywords: acute myeloid leukemia, EQ-5D, health-related quality of life, utility, systematic review, economic analysis, QALY

\section{Introduction}

Acute myeloid leukemia (AML) is an aggressive hematological malignancy that is fatal if left untreated, meriting its designation as a medical emergency. ${ }^{1,2}$ In 2014 , more than 3,000 people were diagnosed with AML in the UK, while around 2,500 patients died due to this condition. ${ }^{3}$ Mortality occurs quickly and is often difficult to prevent. The 5 -year survival rate is $26 \%$ in the US and $17 \%$ in the EU. ${ }^{4}$ Treatment should therefore be initiated shortly after diagnosis, ideally within a matter of days. Even with timely intervention, not all patients achieve remission. As many as $50-70 \%$ of those who do achieve remission following chemotherapy relapse within 3 years. ${ }^{5}$ Bone marrow failure is a defining characteristic of AML. Mortality results predominantly from complications associated with bone marrow failure, such as opportunistic infections or hemorrhage. ${ }^{6}$ 
The treatment pathway for AML can be broken down into three phases: induction chemotherapy, consolidation chemotherapy, and stem cell transplantation (SCT) (Figure 1). Intensive induction chemotherapy is the standard of care for most patients with newly diagnosed AML, aimed at inducing complete remission (CR). ${ }^{1}$ Should the patient achieve CR, they may continue consolidation chemotherapy or receive high-dose chemotherapy conditioning as a bridge to SCT. ${ }^{1}$ Patients often do not reach remission and even if they do, they may still relapse, requiring alternative courses of therapy. ${ }^{5}$ Likewise, successful treatment is not without difficulty: the chemotherapeutic agents used to treat this disease are associated with significant toxicities. ${ }^{7}$ Even SCT is associated with considerable morbidity and mortality, given the high incidence of graft versus host disease (GVHD). ${ }^{8}$

The diagnosis of AML may be very traumatic for a patient who is given little time to adjust before the initiation of aggressive therapy. In this scenario, short-term and long-term well-being are significantly impacted by the initial choice of therapy, ie, the current standard of care in AML management. . $^{910}$

Health-related quality of life (HRQoL) is a measurement of well-being. In one application, it is employed during clinical trials when the impact of an investigational treatment on QoL must be quantified. Clinical trials often consider overall survival or progression-free survival as a primary end point, with HRQoL as a secondary end point. The European Organization for Research and Treatment of Cancer (EORTC) Quality-of-Life Questionnaire Core 30 (QLQ-C30) is a frequently used, non-preference based, cancer-specific questionnaire that assesses HRQoL. ${ }^{11}$

For economic evaluation, many health technology assessment bodies require that effectiveness is expressed in terms of quality-adjusted life-years (QALYs). They also commonly prefer health state utility values (HSUVs) that are determined using the EuroQol five-dimensional (EQ-5D) patient preference questionnaire. ${ }^{12-14}$ QALYs consider both the quantity of survival and its quality. In each disease setting, HSUVs are used to transform the time patients spend in different health states into QALYs.

The EQ-5D was designed to enable the application of cost-effectiveness analysis; patients contribute to definitions of health utility through population surveys and self-reported heath states. EQ-5D scores may then be translated into equivalent QALYs. ${ }^{15,16}$ Patients' preference for one health state over another, as in the EQ-5D scale, dictates their assigned value for that health state. However, the use of EQ-5D in cancer clinical trial data collection is not as widespread as the use of non-preference scales such as the QLQ-C30. The QLQ$\mathrm{C} 30$ consists of 30 questions across five functional scales

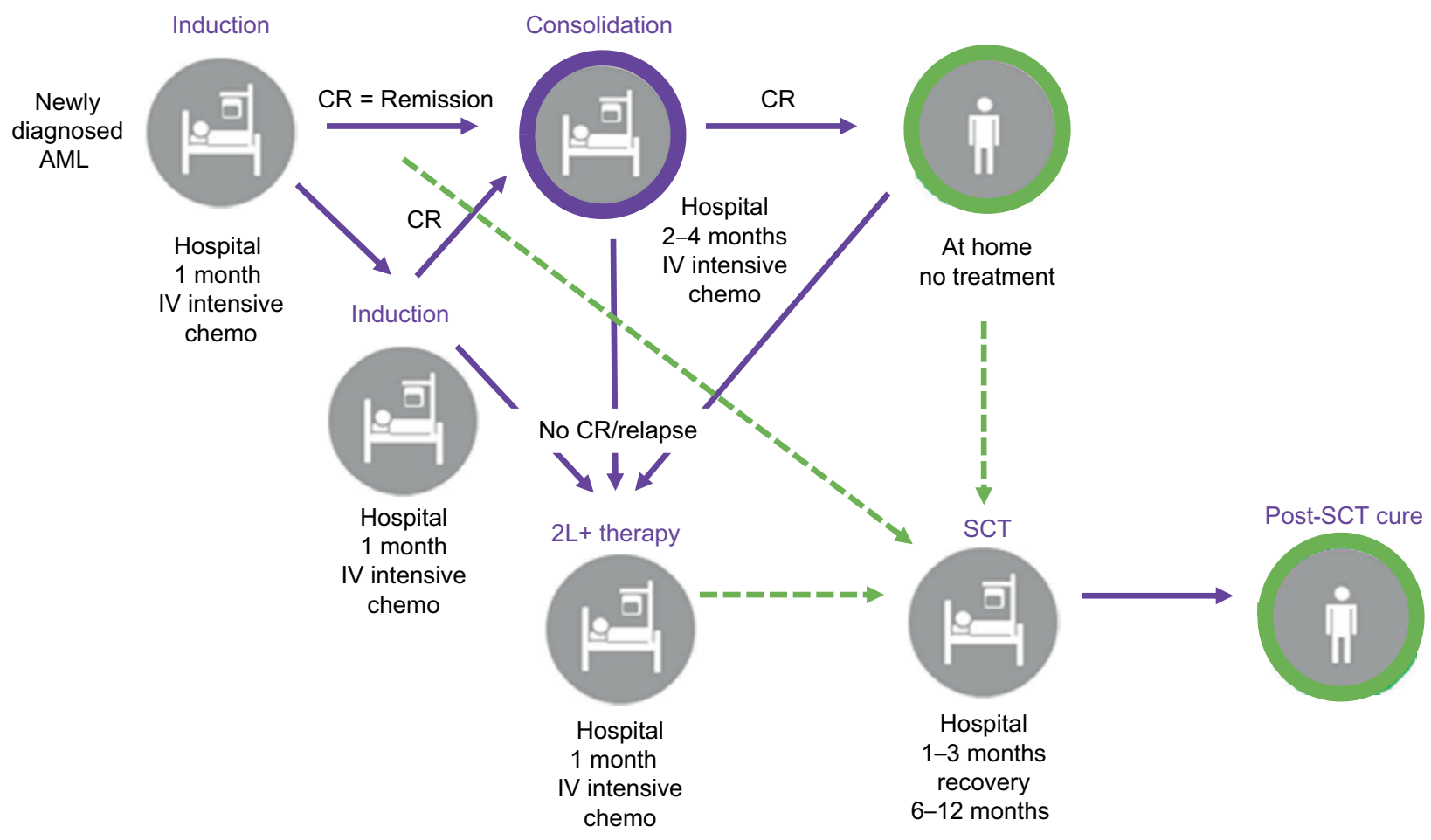

Figure I Treatment pathway for acute myeloid leukemia.

Abbreviations: AML, acute myeloid leukemia; CR, complete remission; IV, intravenous; SCT, stem cell transplantation; 2L, second-line. 
(cognitive, emotional, physical, social, role), nine symptom scales (appetite loss, constipation, diarrhea, dyspnea, fatigue, financial difficulties, insomnia, nausea and vomiting, pain), and one global health state/QoL scale. Higher scores on the functional and global health state scales correspond to better functioning, whereas high symptom scores correspond to worse symptoms. ${ }^{11}$ Where data on health state utilities were not directly collected from patients in clinical trials, utility estimates may be "mapped" from condition-specific HRQoL scales, such as through an algorithmic technique described by Crott et al. ${ }^{17-18}$

Health state utilities for different stages of AML and the toxicities associated with each chemotherapy and SCT regimen are used during the health technology assessment process to estimate the incremental effectiveness of new products versus the standard of care. Most of the previously reported economic analyses have not utilized utilities to describe a range of health states within AML such as pre-relapse versus post-relapse; these studies did not consider the utilities by treatment stage (induction, consolidation, maintenance) or addressed some key health states such as transplantation. ${ }^{19}$ This study aimed to review utility values that may be used in economic evaluations of AML. In the process, it aimed to illustrate and critically discuss challenges in creating a comprehensive set of utility values.

\section{Materials and methods}

A list of relevant health states based on the current consensus in AML treatment was selected and reviewed with UK-based clinical experts experienced in treating AML patients. The health states and their characteristics were defined as part of an unpublished time trade-off (TTO) study. The TTO approach, which seeks to determine the length of lifetime a person would hypothetically sacrifice in exchange for a better health state, has been widely used to obtain health state values. ${ }^{20}$ In the initial stages of TTO process, health state descriptions were developed and validated in collaboration with key opinion leaders and other specialists.

Disease symptoms, adverse events in treatment, and treatment setting were considered as those that may influence the physical, functional, and emotional health of patients.

Relevant health states identified are as follows (Figure 1):

- Newly diagnosed and undergoing induction treatment

- In remission and undergoing consolidation treatment

- Remission post-chemotherapy - long-term follow-up $>1$ year

- SCT procedure

- SCT recovery $<1$ year
- Remission post-SCT, no complications

- Remission post-SCT, GVHD

- Treatment failure/relapse/refractory

A systematic literature review was conducted to identify articles reporting utility data for one or more of the identified health states in AML. In this review, MEDLINE and EMBASE were searched using the Ovid platform, covering nearly 10 years, from January 1, 2006 to November 20, 2016. Systematic reviews and meta-analyses identified were utilized for bibliography searching in order to identify additional relevant studies. In addition, conference abstracts were searched to retrieve studies that had not yet been published as full-text articles and the supplemental results of previously published studies. Abstracts from American Society of Clinical Oncology, European Hematology Society, European Society of Medical Oncology, and American Society of Hematology for the period 2013-2016 were searched. The detailed search strategy is presented in Table S1.

If no studies were identified with data on a specific health state in AML, additional targeted searches were performed to locate studies reporting either utilities or HRQoL related to that specific health state, using a condition that most closely mimics the clinical picture of AML.

Articles were included in the review if they reported utility values for one of the identified health states. Cost effectiveness analyses and health technology assessments were included if utility values were reported. Studies that did not have AML populations and those not reporting utility values were excluded. Only publications written in English and published starting from January 2006 were considered.

Shortlisted articles were initially assessed based on title and abstract. Publications not meeting inclusion criteria were excluded and listed along with the reason for study exclusion. Full-text publications were then retrieved and assessed based on the full text. All steps were conducted by two independent reviewers, and any discrepancies in article selection were reassessed by a third reviewer. After the full-text review, all papers meeting inclusion criteria were retained for data extraction. Papers that were excluded in each step were listed, along with their reason for exclusion. These methods were adapted from the procedure described by the York Centre for Reviews and Dissemination. ${ }^{21}$

Inclusion and exclusion criteria for the systematic review are presented in Table 1.

\section{Health state utility derivations}

Relevant HRQoL data (EORTC QLQ-C30) for patients undergoing SCT were mapped using a previously published 
Table I Study eligibility criteria

\begin{tabular}{|c|c|c|}
\hline Element & Inclusion & Exclusion \\
\hline Patient population & $\begin{array}{l}\text { Patients with acute myeloid leukaemia or myelodysplastic } \\
\text { syndrome }\end{array}$ & Non-human \\
\hline Intervention and comparators & All, including no interventions & \\
\hline Outcome measures & $\begin{array}{l}\text { - Any HRQoL outcomes } \\
\text { - Utilities/disutilities/QALYs for health states or adverse events }\end{array}$ & Any not listed in the inclusion criteria \\
\hline Study design & $\begin{array}{l}\text { - Reports of randomized clinical trials assessing HRQoL } \\
\text { - Development and/or validation of HRQoL measures } \\
\text { - Observational studies measuring PROs } \\
\text { - Retrospective chart audits and database analyses reporting } \\
\text { PROs } \\
\text { - Patient surveys reporting PROs } \\
\text { - Reports of mapping exercises for any outcome measure to } \\
\text { - } \text { utility } \\
\text { - Reports of utility elicitation exercises } \\
\text { - Reports of utility validation exercises } \\
\text { - during the studies } \\
\text { cross-checking only) }\end{array}$ & $\begin{array}{l}\text { - Any not listed in the inclusion criteria } \\
\text { - Reviews } \\
\text { - Editorials } \\
\text { - Notes/Comments/Letters } \\
\text { - Systematic reviews of economic } \\
\text { evaluations (to be used for bibliography } \\
\text { search) }\end{array}$ \\
\hline Restrictions & $\begin{array}{l}\text { - English language } \\
\text { - Year limitation: } 2006 \text { to present }\end{array}$ & Non-English language studies \\
\hline
\end{tabular}

Abbreviations: HRQoL, health-related quality of life; PROs, patient-reported outcomes; QALYs, quality-adjusted life-years.

algorithm to estimate EQ-5D scores from the EORTC QLQC30 scores on the following scales: physical functioning, emotional functioning, social functioning, constipation, diarrhea, pain, and sleep..$^{17,18}$

The algorithm is based on an ordinary least-squares regression algorithm model, which was derived in patients with both the QLQ-C30 questionnaire and the EQ-5D instrument. ${ }^{18}$ In this model, the dependent variable was the calculated overall EQ-5D utility decrement, and the explanatory variables were the calculated QLQ-C30 scores using the following formula:

EQ-5D utility $=0.85927770-$

$0.0069693 *$ (Physical Functioning) -

$0.0087346 *$ (Emotional Functioning $)-$

$0.0039935 *$ (Social Functioning $)+$

$0.0000355 *(\text { Physical Functioning })^{2}+$

$0.0000552 *(\text { Emotional Functioning })^{2}+$

$0.0000290 *(\text { Social Functioning })^{2}+$

$0.0011453 *$ (Constipation) +

$0.0039889 *($ Diarrhea $)+$

$0.0035614 *$ (Pain) -

$0.0003678 *($ Sleep $)-$

$0.0000540 *(\text { Diarrhea })^{2}+$

$0.0000117 *$ Sleep $)^{2}$

In studies where QLQ-C30 values were already mapped to the EQ-5D, the EQ-5D values were utilized.

\section{Results}

The literature search identified 1398 records (Figure 2). A total of 284 full-text articles were retrieved, with 11 of those meeting all inclusion criteria.

The characteristics of the 11 studies are shown in Table 2. ${ }^{19,22,24-30,32}$ Nine studies reported utility values for relevant health states. No studies were identified that reported utility values for patients undergoing SCT treatment. Thus, a targeted search revealed two studies that reported HRQoL (QLQ-C30) for patients undergoing SCT. These were included, with HRQoL data mapped to EQ-5D values as previously described. ${ }^{25,31}$

Table 3 presents the collated utility values, their corresponding AML-relevant health state, and methods of data collection extracted from each study. Many studies measured utility values using the EQ-5D, others employed the QLQC30 and mapping or their utilities were assumptions based on studies of similar conditions, and two studies incorporated utilities based on TTO studies. Although there was some variability in the utilities for each health state, they were relatively similar across the identified studies. The lowest utility values were seen in newly diagnosed patients undergoing induction treatment (range 0.524-0.67) and patients in relapse (range $0.50-0.53$ ). Utility values for patients in remission post-chemotherapy ranged from 0.81 to 0.91 , while values for patients post-SCT ranged from 0.71 to 0.83 . Factors 


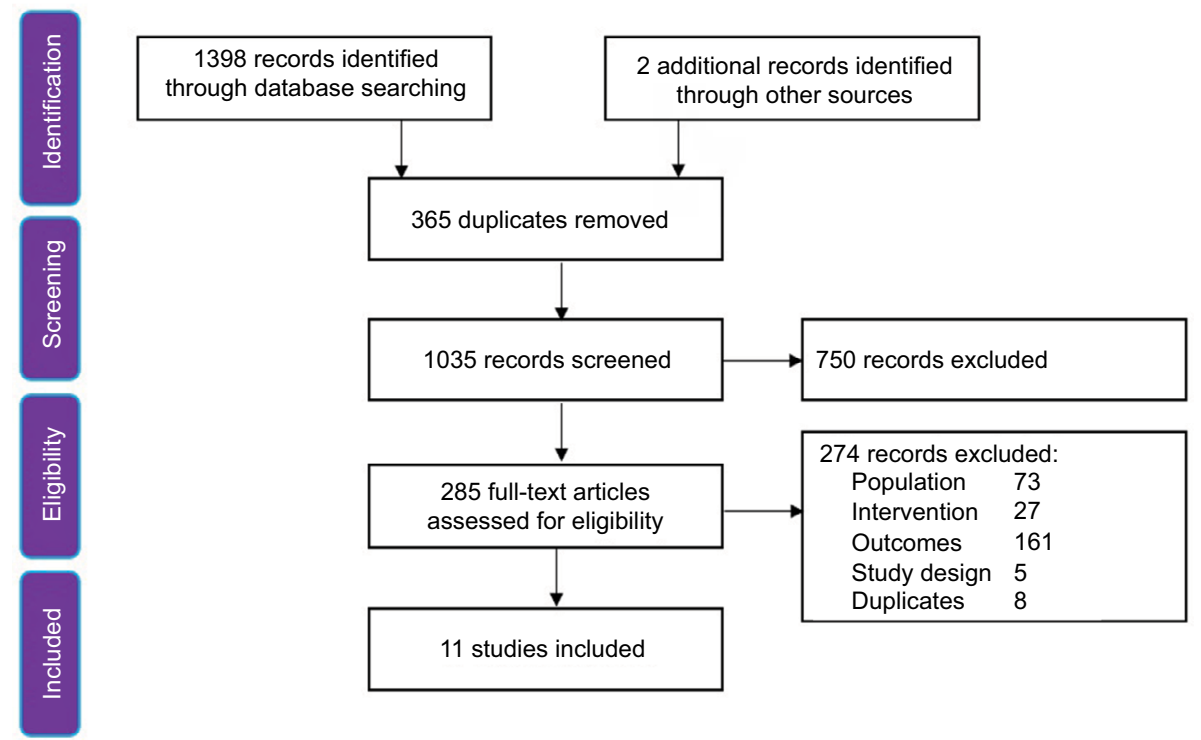

Figure 2 PRISMA flow diagram.

Abbreviation: PRISMA, Preferred Reporting Items for Systematic Reviews and Meta-Analyses.

leading to disutility in remission were age ( 0.61 for patients over 60 years old vs 0.71 for all AML patients), history of prior relapse $(0.78$ with prior relapse vs 0.83 without prior relapse), and GVHD post-SCT ( 0.864 without GVHD vs 0.691 with GVHD).

The utility values can be used in economic models following the AML treatment pathway. Figure 3 illustrates the fluctuation of utility values as they are matched to each health state in the pathway.

\section{Discussion}

Most of the studies utilized data that were collected directly from patients through the EQ-5D questionnaire or the QLQC30 questionnaire. Extrapolation of utilities from other diseases to AML was done for SCT health states (remission and relapse), which identified an area of future research to determine these utilities directly from patients with AML. Based on discussions with the UK-based AML experts, it appears that the utility estimates of the current knowledge base may be fairly representative of health states experienced by AML patients, barring larger and wider-ranging studies on AML utilities in different health states.

The utility values collated show variations in utility across the various health states in AML, with the worst values assigned to both induction and relapse. This finding appears to connote a similarly poor QoL for active AML disease, whether at the beginning of treatment or return of the disease. These low HSUVs in both forms of active AML disease are consistent with findings of a similar systematic review. ${ }^{34}$ More importantly, this stresses the need to focus efforts both on disease reduction and improvement of QoL among patients with active disease.

The transition stage between active disease and remission, ie, the treatment phase, had higher utility values than active disease, which is to be expected given the goal of therapy to reduce disease burden. The values during this stage reflect the complications and side effects that may occur from the therapy itself..$^{35,36}$ This finding is consistent with previous studies on the effect of treatment on both the QoL and functional status of patients with AML. ${ }^{37}$ The persistent deficits in utility values even during remission also illustrate how patients perceive their own well-being during this health state. ${ }^{38}$ As for utility values that exceeded those of the general population, in a TTO approach respondents are not asked to compare their health state to the normal population, but instead to compare their health to the perfect health (utility of 1). As such, the resultant values cannot be compared to the general population. Furthermore, utility scores reported by patients are based on their own individual evaluation of QoL. Thus, patients in remission may report much higher utility scores compared to their diseased health state. The contrast in their QoL between these two health states may lead them to overestimate the QoL of their health state in remission.

EQ-5D utility may depend on the value set used, especially for severe health states. Unfortunately, the identified studies did not report the value sets used in the analyses, which may add uncertainty to the results. Additionally, utility values mapped from the QLQ-C30 instrument should be used with caution 
Table 2 Included studies

\begin{tabular}{|c|c|c|}
\hline Reference & $\begin{array}{l}\text { Type of study/interventions I } \\
\text { study population }\end{array}$ & Utility values \\
\hline \multicolumn{3}{|l|}{ HRQoL studies } \\
\hline \multirow[t]{2}{*}{$(\text { Abstract })^{22}$} & Allogeneic HCT vs Chemo & (complications): 0.67 ; post-chemotherapy overall: 0.70. Scale \\
\hline & Intermediate/unknown-risk AML & version, values sets, and age of respondents not reported \\
\hline \multirow[t]{7}{*}{ Pan et al, $2010^{19}$} & CEA & Transfusion-independent MDS: 0.84 and transfusion-dependent \\
\hline & Decitabine vs BSC & MDS: 0.60 (both from Szende [2009] ${ }^{23}$ TTO study in US, France, \\
\hline & Intermediate/high-risk MDS & Germany, and UK) \\
\hline & & Secondary AML: 0.53 (QLQ-C30 from Alibhai et al \\
\hline & & [2007], ${ }^{35}$ converted to EQ-5D using mapping algorithm by \\
\hline & & Kontodimopoulos et al $[2009]^{38}$ ) \\
\hline & & Scale version, values sets, and age of respondents not reported \\
\hline \multirow[t]{2}{*}{ Leunis et al, $2014^{24}$} & QOL & EQ-5D-5L collected in the Netherlands AML patients, mean \\
\hline & AML survivors post-chemotherapy and HSCT & $\begin{array}{l}\text { AML survivors: } 0.82 \text {; survivors with no relapse: } 0.83 \text {; survivors } \\
\text { after relapse: } 0.78\end{array}$ \\
\hline \multirow[t]{3}{*}{ Slovacek et al, $2007^{25}$} & QOL & EQ-5D-3L collected in Czech AML patients aged 20-69 years, \\
\hline & Autologous HSCT & AML: $0.715 ;$ AML $>60$ years old: 0.61 \\
\hline & AML and malignant Hodgkin's and non- & \\
\hline
\end{tabular}

Hodgkin's lymphoma

QOL

HSCT

Variety of cancers (acute leukemia, CML, solid tumors)

Perić et al, 2016 $6^{27} \quad$ QOL

HSCT patients with and without GVHD

Patients with myeloid malignancies, lymphoid malignancies, and aplastic anemia

Cost-effectiveness studies Levy et al, $2014^{28}$

\section{CEA}

Azacitidine vs conventional chemotherapy (BSC, lowdose chemotherapy, high-dose chemotherapy)

High-risk MDS and low blast AML

CEA

Decitabine vs conventional induction therapy Elderly, newly diagnosed AML

\section{CEA}

Azacitidine vs decitabine

Mixed-risk MDS

Uyl de Groot et al, $1998^{32}$

CEA and QOL

Induction chemo (daunomycin-cytosine arabinoside) + GM-SCF vs induction chemo Elderly AML

\section{CEA}

Lenalidomide

Low/intermediate-risk MDS
Results after mapping to EQ-5D using Crott and Briggs's (2010)18 algorithm (see "Materials and methods" section), before HSCT: 0.826; during hospitalization for HSCT: 0.613 ; up to 6 months after HSCT: 0.8I0; >I year after HSCT: 0.826

QLQ-C30 data collected from HSCT patients 14-70 years old Results after mapping to EQ-5D using Crott and Briggs's (2010) ${ }^{18}$ algorithm (see "Materials and methods" section). Patients without GVHD: 0.864; Patients with GVHD: 0.69।

QLQ-C30 data collected from Croatian HSCT patients with a mean age $=43$ years

AML (>30\% blasts): 0.67

EORTC QLQ-C30 data from clinical trial (Kornblith et al, 2002 ${ }^{35}$ ) was mapped to EQ-5D using published algorithm (McKenzie and van der Pol, 200941). AML utility was assumed to be the same as MDS

QLQ-C30 data were collected in patients with a mean age of 74.8 years

Active AML: 0.524 (use Gidwani et al's [2012] study ${ }^{30}$ ); AML treated with decitabine: $0.7 \mathrm{I}$ (assumption); $\mathrm{AML}$ in remission and on treatment (consolidation and monotherapy): $0.8 \mathrm{I}$ (assumption); AML in remission: 0.91 (based on Goss et al's [2006] study ${ }^{33}$ ) AML: 0.524 (using blast stage of CML, Dalziel et al, 2005'31); remission: 0.9l (based on Goss et al, 2006 study $^{33}$ )

EQ-5D (version not reported) collected in Dutch AML patients $\geq 60$ years old: start of Induction: 64.8/70.6; during hospitalization 53.5/67.I; after hospitalization: 68.0/72.7; 6 months posttreatment: 80.6/84.4; 12 months post-treatment: $74.4 / 75.0$ - with GM-SCF vs without, respectively

TTO study in the US general population, transfusion-dependent MDS: $0.50 ; 50 \%$ reduced transfusion burden: 0.81 ; transfusionindependent MDS: 0.91

Abbreviations: HRQoL, health-related quality of life; HCT, hematopoietic cell transplantation; AML, acute myeloid leukemia; CEA, cost-effectiveness analysis; BSC, best supportive care; MDS, myelodysplastic syndrome; QoL, quality of life; HSCT, hematopoietic stem cell transplantation; CML, chronic myelogenous leukemia; GVHD, graft versus host disease; EQ-5D, EuroQol five-dimensional; SCT, stem cell transplantation; QLQ-C30, Quality of Life Questionnaire Core 30; EQ-5D-5L, EuroQoL 5 dimensions 5-level; EQ-5D-3L, EuroQoL 5 dimensions 3-level; EORTC, European Organization for Research and Treatment of Cancer; GM-SCF, granulocyte-macrophage colonystimulating factor; TTO, time trade-off. 
Table 3 Utility values for reported health states in acute myeloid leukemia

\begin{tabular}{|c|c|c|c|c|}
\hline Reported health state & AML-relevant health state & $\begin{array}{l}\text { Utility value } \\
\text { point estimate }\end{array}$ & Reference & Data source \\
\hline Active AML & Induction & 0.524 & Gidwani et al, $2012^{30}$ & Assumption based on CML \\
\hline Newly diagnosed & Induction & 0.67 & Levy et al, $2014^{28}$ & Mapped from QLQ-C30 \\
\hline Induction treatment & Induction & 0.648 & Uyl-de-Groot et al, $1998^{32}$ & Measured EQ-5D \\
\hline AML treated with decitabine & Consolidation & 0.71 & Batty et al, $2014^{29}$ & Assumption, calculated \\
\hline After initial hospitalization & Consolidation & 0.68 & Uyl-de-Groot et al, $1998^{32}$ & Measured EQ-5D \\
\hline In remission and on treatment & Maintenance & 0.81 & Batty et al, $2014^{29}$ & Assumption, calculated \\
\hline Six months post-treatment & Remission & 0.806 & Uyl-de-Groot et al, $1998^{32}$ & Measured EQ-5D \\
\hline Transfusion-independent MDS & Remission & 0.84 & Pan et al, $2010^{19}$ & TTO Study in MDS \\
\hline Transfusion-independent MDS & Remission & 0.91 & Goss et al, $2006^{33}$ & TTO study in MDS \\
\hline AML survivors without relapse & Remission, post-IL & 0.83 & Leunis et al, $2014^{24}$ & Measured EQ-5D \\
\hline AML survivors post-relapse & Remission post-relapse & 0.78 & Leunis et al, $2014^{24}$ & Measured EQ-5D \\
\hline Remission prior to SCT & Remission post-chemotherapy & 0.826 & Grulke et al, $2012^{26}$ & Mapped from QLQ-C30 \\
\hline Long-term post-chemo & Long-term remission & 0.70 & Kurosawa et al, $2014^{22}$ & Measured EQ-5D \\
\hline Twelve months post-treatment & Long-term remission & 0.744 & Uyl-de-Groot et al, $1998^{32}$ & Measured EQ-5D \\
\hline During SCT treatment & SCT treatment & 0.613 & Grulke et al, $2012^{26}$ & Mapped from QLQ-C30 \\
\hline SCT recovery: 6-12 months & SCT recovery & 0.810 & Grulke et al, $2012^{26}$ & Mapped from QLQ-C30 \\
\hline Post-SCT: $>12$ months & Post-SCT & 0.826 & Grulke et al, $2012^{26}$ & Mapped from QLQ-C30 \\
\hline Post-SCT & Remission post-SCT & 0.71 & Slovacek et al, $2007^{25}$ & Measured EQ-5D \\
\hline Post-SCT $>60$ years old & Remission post-SCT, elderly & 0.61 & Slovacek et al, $2007^{25}$ & Measured EQ-5D \\
\hline Post-SCT & Post-SCT & 0.74 & Kurosawa et al, $2014^{22}$ & Measured EQ-5D \\
\hline Post-SCT without GVHD & Post-SCT without GVHD & 0.864 & Perić et al, $2016^{27}$ & Mapped from QLQ-C30 \\
\hline Post-SCT with GVHD & Post-SCT with GVHD & 0.691 & Perić et al, $2016^{27}$ & Mapped from QLQ-C30 \\
\hline Post-SCT with GVHD & Post-SCT with GVHD & 0.67 & Kurosawa et al, $2014^{22}$ & Measured EQ-5D \\
\hline Secondary AML & Relapse & 0.53 & Pan et al, $2010^{19}$ & Mapped from QLQ-C30 \\
\hline Transfusion-dependent MDS & Relapse & 0.50 & Goss et al, $2006^{33}$ & TTO Study in MDS \\
\hline
\end{tabular}

Abbreviations: AML, acute myeloid leukemia; MDS, myelodysplastic syndromes; SCT, stem cell transplantation; GVHD, graft versus host disease; CLL, chronic lymphocytic leukemia; TTO, time trade-off; QLQ-C30, Quality of Life Questionnaire Core 30; EQ 5D, EuroQol five-dimensional; IL, first-line.

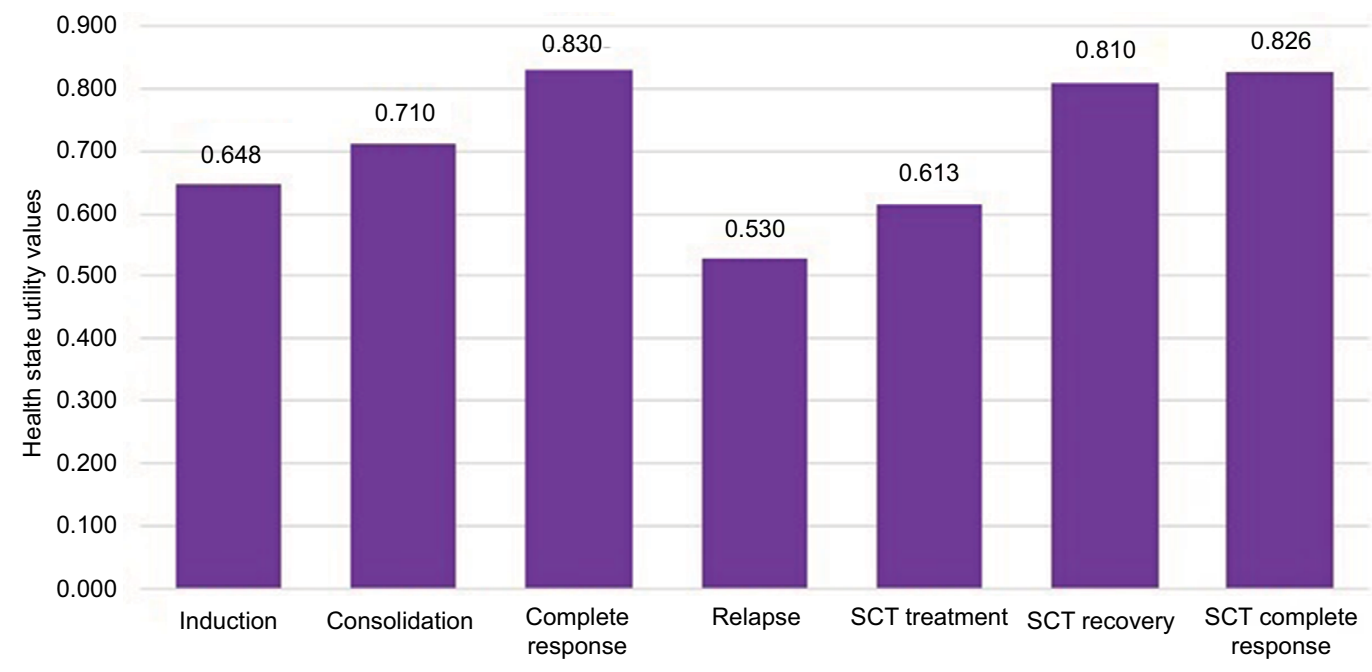

Figure 3 Health state utility values for acute myeloid leukemia. Abbreviation: SCT, stem cell transplantation.

because of the derivative nature of the values generated ${ }^{37}$ While the validity of mapping techniques has been tested against EQ-5D and other preference-based instruments, such as short form 6 dimensions (SF-6D) and 15 dimensions (SF-15D), it is important to note that primary data for utilities taken directly from patients would still hold priority over these derived values. ${ }^{38,39}$ As experience grows with the use of these instruments and their mapping into QALYs, studies collating and validating this application may be beneficial for future economic analyses, especially when there is a lack of primary studies on utilities focusing on particular diseases such as AML. ${ }^{39-42}$

In our selection of final utility values recommended for use in health economic evaluations, we prioritized values collected directly from patients to those mapped. We, how- 
ever, made an exception for the utility values related to SCT. Although Kurosawa et al's publication reported EQ-5D values collected directly from patients, the validity of this research is diminished since it was never published in a peer-reviewed journal. ${ }^{22}$ Furthermore, the values reported by Kurosawa et al were considerably lower as compared to other studies, such as those of Uyl-de Groot et al and Leunis et al. ${ }^{31,24}$ On the other hand, the mapped values using Grulke et al for remission prior to SCT were more consistent with values reported by other studies. ${ }^{27}$ The consistently lower HSUVs reported in the Kurosawa et al's study compared to other studies is the basis for the selection of the HSUVs mapped from Grulke et al. Although the use of mapping applied to mean values versus individual patient level adds potential weakness to resultant utility values, the HSUVs derived from Grulke et al provided a more clinically plausible progression in the improvement of QoL with successful treatment (ie, with SCT), as depicted in Figure 3. Selecting the HSUV provided by Kurosawa et al would have portrayed a significant decline in HSUV following SCT not compatible with known clinical course and prognosis of patients in that health state..$^{7,43-46}$

Overall, this study uncovered a common issue when searching for utility values for rare diseases, such as AML. Studies on rare diseases are relatively rare in themselves and require considerable resources to find.$^{47}$ In this study, only 11 have met the inclusion criteria, with many studies excluded mainly because they did not deal with acute hematologic malignancy, and because they did not examine QoL or utility outcomes. One recommendation is to institute a comprehensive policy covering the registration and monitoring of the health status of patients with rare diseases, including their treatment regimens, clinical outcomes, and QoL. ${ }^{48}$ With such broad remit, multiple studies may be generated from these datasets and registries, so-called "real-world evidence", enabling the creation of a more complete picture of the disease process, its treatment, and experience living with the disease. TTO studies may likewise be performed to extract further information from existing registries and research., ${ }^{9,49,50}$

More importantly, the determination of HSUVs for rare diseases is particularly challenging, especially since the populations used may be smaller than usual (resulting in wider ranges) and the methodology of determining HSUVs may vary considerably as well. ${ }^{50}$ Establishing a set of recommendations to standardize HSUV determination across different diseases and contexts may be valuable in the long term.

\section{Conclusion}

Here we present a broad summary of the available utility scores in AML. There are relatively few methodologically robust HSUVs that can be directly used in economic evaluations concerned with AML. Careful interpretation of published values is advised considering significant differences in methodologies as well as patient population inclusion and size. There is a need to develop new HSUVs with larger-sized studies which improve on those currently available, either by utilizing TTO studies or by making greater use of conditionspecific data and further use of mapping algorithms.

\section{Acknowledgment}

This study was sponsored by Novartis. We would like to acknowledge Jaclyn Hearnden for her assistance in preparing the manuscript. Parts of this study were previously published in an abstract submitted to the 2017 European Hematology Association Congress.

\section{Author contributions}

AF, GT, SP, and PSB made substantial contributions to the conception and design, analyzed and interpreted the data, and critically revised the article. MD acquired and analyzed the data, and contributed to the revision of the manuscript. APJR contributed to data interpretation and drafting of the article. All authors contributed toward data analysis, drafting and revising the paper and agree to be accountable for all aspects of the work.

\section{Disclosure}

The authors report no conflicts of interest in this work.

\section{References}

1. Fathi AT, Chen YB. Treatment of FLT3-ITD acute myeloid leukemia. Am J Blood Res. 2011;1(2):175-189.

2. Sekeres MA, Elson P, Kalaycio ME, et al. Time from diagnosis to treatment initiation predicts survival in younger, but not older, acute myeloid leukemia patients. Blood. 2009;113(1):28-36.

3. Acute myeloid leukaemia (AML) statistics; 2014. Available from: http://www.cancerresearchuk.org/health-professional/cancer-statistics/ statistics-by-cancer-type/leukaemia-aml. Accessed May 12, 2017.

4. Maynadie M, De Angelis R, Marcos-Gragera R, et al. Survival of European patients diagnosed with myeloid malignancies: a HAEMACARE study. Haematologica. 2013;98(2):230-238.

5. Dohner H, Estey EH, Amadori S, et al. Diagnosis and management of acute myeloid leukemia in adults: recommendations from an international expert panel, on behalf of the European LeukemiaNet. Blood. 2010;115(3):453-474.

6. Deschler B, Lubbert M. Acute myeloid leukemia: epidemiology and etiology. Cancer. 2006;107(9):2099-2107.

7. Dohner H, Estey E, Grimwade D, et al. Diagnosis and management of AML in adults: 2017 ELN recommendations from an international expert panel. Blood. 2017;129(4):424-447.

8. Jacobsohn DA, Vogelsang GB. Acute graft versus host disease. Orphanet J Rare Dis. 2007;2:35.

9. Cannella L, Caocci G, Jacobs M, Vignetti M, Mandelli F, Efficace F. Health-related quality of life and symptom assessment in randomized controlled trials of patients with leukemia and myelodysplastic syndromes: What have we learned? Crit Rev Oncol Hematol. 2015;96(3): $542-554$. 
10. Redaelli A, Stephens JM, Brandt S, Botteman MF, Pashos CL. Short- and long-term effects of acute myeloid leukemia on patient health-related quality of life. Cancer Treat Rev. 2004;30(1):103-117.

11. Fayers PM, Aaronson NK, Bjordal K, Groenvold M, Curran D, Bottomley A, on behalf of the EORTC Quality of Life Group. The EORTC QLQ-C30 Scoring Manual. 3rd ed. Brussels: The European Organisation for Research and Treatment of Cancer; 2001.

12. Single Technology Appraisal (STA). Specification for Manufacturer/ Sponsor Submission. Rotterdam, The Netherlands: National Institute for Health and Clinical Excellence; 2012.

13. Brazier J, Longworth L. NICE DSU Technical Support Document 8: An Introduction to the Measurement and Valuation of Health for NICE Submissions. London: National Institute for Health and Care Excellence (NICE); 2011.

14. Papaioannou D, Brazier J, Paisley S. NICE DSU Technical Support Document 9: The Identification, Review and Synthesis of Health State Utility Values from the Literature. London: National Institute for Health and Care Excellence (NICE); 2010.

15. Bansback N, Harrison M, Brazier J, et al. Health state utility values: a description of their development and application for rheumatic diseases. Arthritis Rheum. 2008;59(7):1018-1026.

16. Devlin NJ, Brooks R. EQ-5D and the EuroQol Group: past, present and future. Appl Health Econ Health Policy. 2017;15(2):127-137.

17. Crott R, Versteegh M, Uyl-de-Groot C. An assessment of the external validity of mapping QLQ-C30 to EQ-5D preferences. Qual Life Res. 2013;22(5):1045-1054.

18. Crott R, Briggs A. Mapping the QLQ-C30 quality of life cancer questionnaire to EQ-5D patient preferences. Eur J Health Econ 2010;11(4):427-434.

19. Pan F, Peng S, Fleurence R, Linnehan JE, Knopf K, Kim E. Economic analysis of decitabine versus best supportive care in the treatment of intermediate- and high-risk myelodysplastic syndromes from a US payer perspective. Clin Ther. 2010;32(14):2444-2456.

20. Morimoto T, Fukui T. Utilities measured by rating scale, time trade-off, and standard gamble: review and reference for health care professionals. J Epidemiol. 2002;12(2):160-178.

21. Systematic Reviews: CRD's Guidance for Undertaking Reviews in Health Care. York, UK: Centre for Reviews and Dissemination, University of York; 2009.

22. Kurosawa S, Yamaguchi H, Yamaguchi T, et al. decision analysis of allogeneic hematopoietic stem cell transplantation versus chemotherapy in cytogenetically standard-risk acute myeloid leukemia in first complete remission: the impact of FLT3-ITD profile. Blood. 2014;124(21):1221-1221.

23. Szende A, Schaefer C, Goss TF, Heptinstall K, Knight R, Lübbert M, et al. Valuation of transfusion-free living in MDS: results of health utility interviews with patients. Health Qual Life Outcomes. 2009;7:81.

24. Leunis A, Redekop WK, Uyl-de Groot CA, Lowenberg B. Impaired health-related quality of life in acute myeloid leukemia survivors: a single-center study. Eur J Haematol. 2014;93(3):198-206.

25. Slovacek L, Slovackova B, Jebavy L, Macingova Z. Psychosocial, health and demographic characteristics of quality of life among patients with acute myeloid leukemia and malignant lymphoma who underwent autologous hematopoietic stem cell transplantation. Sao Paulo Med J. 2007;125(6):359-361.

26. Grulke N, Albani C, Bailer H. Quality of life in patients before and after haematopoietic stem cell transplantation measured with the European Organization for Research and Treatment of Cancer (EORTC) Quality of Life Core Questionnaire QLQ-C30. Bone Marrow Transplant. 2012;47(4):473-482.

27. Peric Z, Desnica L, Durakovic N, et al. Which questionnaires should we use to evaluate quality of life in patients with chronic graft-vs-host disease? Croat Med J. 2016;57(1):6-15.

28. Levy AR, Zou D, Risebrough N, Buckstein R, Kim T, Brereton N. Costeffectiveness in Canada of azacitidine for the treatment of higher-risk myelodysplastic syndromes. Curr Oncol. 2014;21(1):e29-40.

29. Batty N, Yin Y, Wetzler M. Decitabine is more cost effective than cytarabine and daunorubicin in elderly acute myeloid leukemia patients. J Cancer Res Ther. 2014;2(4):68-73.
30. Gidwani R, Khan ZM, Fenaux P, Beach CL, Pashos CL. A cost-effectiveness analysis of using azacitidine vs. decitabine in treating patients with myelodysplastic syndromes. J Med Econ. 2012;15(1):145-154.

31. Dalziel K, Round A, Garside R, Stein K. Cost effectiveness of imatinib compared with interferon-alpha or hydroxycarbamide for first-line treatment of chronic myeloid leukaemia. Pharmacoeconomics. 2005;23(5):515-526.

32. Uyl-de Groot CA, Lowenberg B, Vellenga E, Suciu S, Willemze R, Rutten FF. Cost-effectiveness and quality-of-life assessment of GM-CSF as an adjunct to intensive remission induction chemotherapy in elderly patients with acute myeloid leukemia. Br J Haematol. 1998;100(4):629-636.

33. Goss TF, Szende A, Schaefer C, et al. Cost effectiveness of lenalidomide in the treatment of transfusion-dependent myelodysplastic syndromes in the United States. Cancer Control. 2006;13(Suppl):17-25.

34. Korol EE, Wang S, Johnston K, Ravandi-Kashani F, Levis M, van Nooten F. Health-related quality of life of patients with acute myeloid leukemia: a systematic literature review. Oncol Ther. 2017;5(1):1-16.

35. Kornblith AB, Herndon JE, 2nd, Silverman LR, et al. Impact of azacytidine on the quality of life of patients with myelodysplastic syndrome treated in a randomized phase III trial: a Cancer and Leukemia Group B study. J Clin Oncol. 2002;20(10):2441-2452.

36. Schumacher A, Kessler T, Buchner T, Wewers D, van de Loo J. Quality of life in adult patients with acute myeloid leukemia receiving intensive and prolonged chemotherapy - a longitudinal study. Leukemia. 1998;12(4):586-592.

37. Alibhai SM, Leach M, Kermalli H, et al. The impact of acute myeloid leukemia and its treatment on quality of life and functional status in older adults. Crit Rev Oncol Hematol. 2007;64(1):19-30.

38. Tinsley SM, Small BJ, Lancet JE, et al. What is the impact on quality of life of various treatments in acute myeloid leukemia and high-risk myelodysplastic syndrome? Rethinking the goals. Blood. 2015;126(23):2112-2112.

39. Crott R. Mapping algorithms from QLQ-C30 to EQ-5D utilities: no firm ground to stand on yet. Expert Rev Pharmacoecon Outcomes Res. 2014;14(4):569-576.

40. Kontodimopoulos N, Aletras VH, Paliouras D, Niakas D. Mapping the cancer-specific EORTC QLQ-C30 to the preference-based EQ-5D, SF-6D, and 15D instruments. Value Health. 2009;12(8):1151-1157.

41. McKenzie L, van der Pol M. Mapping the EORTC QLQ C-30 onto the EQ-5D instrument: the potential to estimate QALYs without generic preference data. Value Health. 2009;12(1):167-171.

42. Dakin H. Review of studies mapping from quality of life or clinical measures to EQ-5D: an online database. Health Qual Life Outcomes. $2013 ; 11: 151$.

43. Eapen M, Logan BR, Appelbaum FR, et al. Long-term survival after transplantation of unrelated donor peripheral blood or bone marrow hematopoietic cells for hematologic malignancy. Biol Blood Marrow Transplant. 2015;21(1):55-59.

44. Kanate AS, Pasquini MC, Hari PN, Hamadani M. Allogeneic hematopoietic cell transplant for acute myeloid leukemia: current state in 2013 and future directions. World J Stem Cells. 2014;6(2):69-81.

45. Parmesar K, Raj K. Haploidentical stem cell transplantation in adult haematological malignancies. Adv Hematol. 2016;2016:3905907.

46. Majhail NS, Bajorunaite R, Lazarus HM, et al. High probability of long-term survival in 2-year survivors of autologous hematopoietic cell transplantation for AML in first or second CR. Bone Marrow Transplant. 2011;46(3):385-392.

47. Patients'Priorities and Needs for Rare Disease Research 2014-2020. Paris: EURORDIS - Rare Diseases Europe; 2014. Available from: https://www.eurordis.org/sites/default/files/publications/what_ how\%20_are_disease_research_0.pdf. Accessed December 04, 2017.

48. Dharssi S, Wong-Rieger D, Harold M, Terry S. Review of 11 national policies for rare diseases in the context of key patient needs. Orphanet J Rare Dis. 2017;12(1):63.

49. Buckley SA, Lee SJ, Walter RB. Measuring quality of life in acute myeloid leukemia: limitations and future directions. Expert Rev Hematol. 2016;9(9):821-823.

50. Papaioannou D, Brazier J, Paisley S. Systematic searching and selection of health state utility values from the literature. Value Health 2013;16(4):686-695. 


\section{Supplementary material}

Table SI Search strategy

\begin{tabular}{|c|c|c|}
\hline I & Acute myeloid leukemia.ti,ab. & 60263 \\
\hline 2 & myelodysplastic syndrome.ti,ab. & 23550 \\
\hline 3 & I or 2 & 77788 \\
\hline 4 & quality adjusted life.ti,ab. & 25093 \\
\hline 5 & qaly\$.ti,ab. & 23165 \\
\hline 6 & qol.ti,ab. & 86692 \\
\hline 7 & quality of life.ti,ab. & 564529 \\
\hline 8 & exp "quality of life"/ & 579230 \\
\hline 9 & exp Quality-Adjusted Life Years/ & 33606 \\
\hline 10 & Quality adjusted life year\$.ti,ab. & 24190 \\
\hline II & Health-related quality of life.ti,ab. & 84721 \\
\hline 12 & hrqol.ti,ab. & 31994 \\
\hline 13 & hrql.ti,ab. & 8328 \\
\hline 14 & health utilit\$ index.ti,ab. & 1939 \\
\hline 15 & HUl.ti,ab. & 2335 \\
\hline 16 & health utilit\$.ti,ab. & 4305 \\
\hline 17 & (hui or huil or hui2 or hui3).ti,ab. & 3024 \\
\hline 18 & disutil\$.ti,ab. & 970 \\
\hline 19 & utility.ti,ab. & 374317 \\
\hline 20 & utility analysis.ti,ab. & 5699 \\
\hline 21 & assessment of quality of life.ti,ab. & 4109 \\
\hline 22 & time trade off.ti,ab. & 2484 \\
\hline 23 & TTO.ti,ab. & 2226 \\
\hline 24 & euroqol.ti,ab. & 10167 \\
\hline 25 & (euroqol $5 \mathrm{~d}$ or EQ-5D or eq-5d or euroqol).ti,ab. & 21006 \\
\hline 26 & eq $\$ 5$ d.ti,ab. & 1648 \\
\hline 27 & (short form $6 \mathrm{~d}$ or shortform $6 \mathrm{~d}$ or sf6d or sf-6d or sf $6 \mathrm{~d}$ ).ti,ab. & 1978 \\
\hline 28 & $\begin{array}{l}4 \text { or } 5 \text { or } 6 \text { or } 7 \text { or } 8 \text { or } 9 \text { or } 10 \text { or } 11 \text { or } 12 \text { or } 13 \text { or } 14 \text { or } 15 \text { or } 16 \text { or } 17 \text { or } 18 \text { or } 19 \text { or } 20 \text { or } 21 \text { or } \\
22 \text { or } 23 \text { or } 24 \text { or } 25 \text { or } 26 \text { or } 27\end{array}$ & 1123462 \\
\hline 29 & cost-effectiveness.ti,ab. & 128870 \\
\hline 30 & technology assessment.ti,ab & 11675 \\
\hline 31 & pharmacoeconomic $\$ . t i, a b$. & 10976 \\
\hline 32 & 28 or 29 or 30 or 31 & 1223333 \\
\hline 33 & 3 and 32 & 2087 \\
\hline 34 & limit 33 to human & 1860 \\
\hline 35 & limit 34 to english language & 1752 \\
\hline 36 & limit 35 to $y r=" 2006$-Current" & 1398 \\
\hline 37 & remove duplicates from 36 & 1033 \\
\hline
\end{tabular}

Abbreviations: EQ-5D, EuroQol five-dimensional; HUI, health utility index; TTO, time trade-off.

\section{Publish your work in this journal}

ClinicoEconomics and Outcomes Research is an international, peerreviewed open-access journal focusing on health technology assessment, pharmacoeconomics and outcomes research in the areas of diagnosis, medical devices, and clinical, surgical and pharmacological intervention. The economic impact of health policy and health systems organization also constitute important areas of coverage. The manuscript management system is completely online and includes a very quick and fair peer-review system, which is all easy to use. Visit http://www.dovepress.com/testimonials.php to read real quotes from published authors. 